\title{
Des concepts aux indicateurs du développement durable: multidimensionnalité et responsabilisation
}

\section{Gilles Allaire et Thierry Dupeuble}

\section{OpenEdition}

1 Journals

\section{Édition électronique}

URL : https://journals.openedition.org/developpementdurable/678

DOI : 10.4000/developpementdurable.678

ISSN : 1772-9971

Éditeur

Association DD\&T

\section{Référence électronique}

Gilles Allaire et Thierry Dupeuble, « Des concepts aux indicateurs du développement durable: multidimensionnalité et responsabilisation », Développement durable et territoires [En ligne], Varia (2004-2010), mis en ligne le 21 janvier 2004, consulté le 21 septembre 2021. URL : http:// journals.openedition.org/developpementdurable/678 ; DOI : https://doi.org/10.4000/ developpementdurable.678

Ce document a été généré automatiquement le 21 septembre 2021.

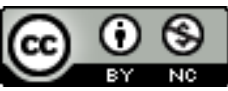

Développement Durable et Territoires est mis à disposition selon les termes de la licence Creative Commons Attribution - Pas d'Utilisation Commerciale 4.0 International. 


\title{
Des concepts aux indicateurs $d u$ développement durable: multidimensionnalité et responsabilisation
}

\author{
Gilles Allaire et Thierry Dupeuble
}

1 La question des indicateurs du développement durable est posée depuis que la définition qu'en a proposé Gro Bruntland dans son rapport de 1989 «Notre avenir à tous» a été officiellement consacrée dans le cadre du Sommet de la Terre à Rio en 1992: «Satisfaire les besoins des générations actuelles sans compromettre la capacité des générations futures à satisfaire les leurs». En effet, définie dans cette globalité, la notion de développement durable peine à être opérationnelle. Quels contenus donner au développement durable en termes techniques, organisationnels et de dispositifs publics et quels indicateurs pour les instrumenter sont deux questions majeures pour donner au concept une réalité pratique. Les Nations Unies ont fait des propositions générales d'indicateurs en 1996; l'IFEN (Institut français de l'environnement) a été chargé d'en tester la faisabilité dans le cadre français ; toutefois ces indicateurs ne sont pas encore chiffrés (IFEN, 2001). S'agissant de l'agriculture (le domaine abordé dans ce texte), ces questions ont mobilisé, depuis peu, chercheurs et acteurs; les propositions de définitions et d'indicateurs se sont multipliées en quelques années, mais on ne dispose pas encore aujourd'hui, en ce domaine non plus, d'indicateurs arrêtés. De plus, de nombreuses questions de recherche sur les interdépendances dans le temps et dans l'espace entre activités et entre territoires apparaissent en amont de la construction de ces indicateurs.

2 La thématique du développement durable, qui est loin d'être établie, n'est pas uniquement un nouvel objet de recherche. Elle se présente également comme une nouvelle gouvernance de la recherche, où la co-construction des objets de recherche joue un rôle fondamental ${ }^{1}$. De plus, la notion de développement durable s'inscrit désormais dans une stratégie de l'Etat (voir la création du Comité interministériel pour le 
développement durable, janvier 2003). Ainsi, le développement durable devient l'aune à laquelle les politiques publiques doivent être évaluées, ce qui multiplie l'intérêt pour des indicateurs. C'est notamment le cas des politiques agricoles et rurales. En effet, l'agriculture, plus que toute autre activité, dépend du renouvellement des ressources naturelles qu'elle valorise, c'est d'ailleurs là sa raison d'être. Elle est également l'un des domaines particulièrement visés par les critiques au nom de l'environnement. Cet article analyse le problème de la construction d'indicateurs du développement durable, du niveau de l'évaluation des activités des exploitations agricoles à celui des politiques publiques à enjeux territoriaux ${ }^{2}$.

3 C'est par l'explicitation de dimensions constitutives de la durabilité que la notion a commencé d'être précisée: le développement durable se doit d'être économiquement viable (satisfaction des besoins d'une génération...), socialement équitable (...dans une solidarité horizontale...) et écologiquement reproductible (...et une solidarité verticale i.e. intergénérationnelle-). Ce triptyque a parfois évolué vers une version quadridimensionnelle: viabilité, vivabilité, reproductibilité, transmissibilité (Landais, 1998); économie, environnement, équité, éthique (Lepage, 1997), mais sans que soit véritablement modifiée la décomposition de la notion. Ceci a ouvert la voie à des approches analytiques opérationnelles fondées sur la construction d'une batterie d'indicateurs par dimension. Des associations -Solagro (Dialecte), le Réseau agriculture Durable-, le Ministère de l'Agriculture -Bergerie nationale (Idea)-, des syndicats Confédération paysanne-, ou encore des plates-formes institutionnelles -Farre ${ }^{3}$-, ont tour à tour élaboré leurs indicateurs. Ces grilles témoignent de divergences notables sur l'importance qu'elles accordent à telle ou telle dimension et donc sur le contenu ainsi donné à la durabilité.

4 Au-delà de leurs divergences, les grilles d'évaluation que nous avons évoquées ont en commun d'être applicables au niveau de l'exploitation agricole. C'est donc l'activité d'une unité de production, prise dans sa singularité, qui fait l'objet d'une évaluation du point de vue de la durabilité. Cette perspective est celle d'une appréciation de la participation d'une activité donnée ou d'un acteur en particulier à un développement qui serait durable. C'est aussi la perspective des "Agendas 21", où le développement durable est l'affaire des acteurs citoyens. C'est bien dans un tel esprit que se sont constitués des réseaux professionnels "développement durable", qu'il s'agisse du réseau «Agriculture durable» impulsé par les Civam $^{4}$ de l'Ouest, de Farre ou encore du réseau "Démonstration» ${ }^{5}$ de l'enseignement agricole. Dans cet esprit, les diagnostics d'exploitation participent de l'auto-évaluation et de la responsabilisation ${ }^{6}$. Toutefois, chaque réseau mène aussi une bataille pour l'institutionnalisation et la normalisation de ses propositions, sous forme de reconnaissance de bonnes pratiques et d'indicateurs pertinents, et, ainsi, pour faire reconnaître son engagement dans des programmes publics. Parallèlement émerge la question de l'évaluation des programmes publics au regard de l'engagement qu'ils permettent dans des dynamiques de développement durable et donc la recherche d'indicateurs caractérisant les dynamiques de développement elles-mêmes.

$5 \quad$ Les diagnostics des activités au niveau des exploitations ne se limitent pas au seul espace constitué par l'ensemble des parcelles cadastrées, mais concernent plus largement le cadre agronomique ou agro-écologique de ces activités. Les indicateurs tentent de saisir la contribution de chacune d'entre elles à la durabilité du territoire dans lequel elles sont insérées. On pourrait alors être tenté d'agréger ces observations pour mettre en œuvre 
des diagnostics de territoire. Pour certains indicateurs, c'est localement possible. Mais, au niveau local, on ne peut pas saisir certains effets d'interdépendance entre exploitations et entre territoires (les flux économiques ne permettent d'en saisir qu'une partie). Ainsi, par exemple, on pourra réaliser, à l'échelle d'une portion de bassin versant, un bilan de fertilisation «agrégé» à partir des bilans individuels réalisés sur chacune des exploitations concernées. La mise en débat des résultats d'une telle démarche peut permettre de passer à une réflexion et à une responsabilisation de groupe et donc à des initiatives locales individuelles et collectives destinées à «améliorer la situation» (à l'instar des "analyses de groupe" des comptabilités économiques réalisées par les conseillers agricoles, il y a une trentaine d'années, qui permettaient à chaque agriculteur de situer sa «performance» au sein du groupe et prendre conscience des marges de progression). Mais, cette seule échelle d'appréhension ne convient pas pour évaluer la durabilité de l'activité agricole plus globalement. Une évaluation globale ne saurait procéder d'une agrégation d'évaluations élémentaires locales, laquelle ne permet pas de saisir les effets d'interdépendance entre exploitations et entre territoires. Il en est ainsi par exemple de la biodiversité ou du paysage. Parmi les critères mobilisés par les différentes grilles d'évaluation, le nombre de mètres linéaires de haies est quasi systématiquement repris. Plus ce nombre est élevé, plus l'exploitation est durable au sens où sa contribution tant au paysage qu'à la biodiversité apparaît appréciable. Cela signifie implicitement que si l'on passe du niveau individuel, l'exploitation, au niveau supérieur, le territoire local, il y a un simple effet d'additionnalité. Or rien n'est moins vrai. Le passage de la haie au paysage bocager suppose une coordination qui n'a rien d'une simple addition. Il en est de même pour la contribution à la biodiversité.

6 Comme le fait remarquer Landais (1999), la prise en compte par la définition du développement durable d'une part des besoins des générations futures, et d'autre part des problèmes environnementaux d'ordre planétaire (par exemple, l'effet de serre), implique une réflexion intégrant des échelles de temps et d'espace inhabituelles dans la sphère de la recherche appliquée à l'agriculture. L'évaluation des pratiques agricoles se heurte ainsi à «de très grandes difficultés méthodologiques et métrologiques» (Landais, 1999). Il ne s'agit plus seulement d'évaluer les effets de telle ou telle pratique sur des objets directement visés ici et maintenant mais aussi les conséquences ailleurs et plus tard sur le contexte de ces objets. Au fond, ce qui est en question est la difficulté d'élaboration d'un référentiel à partir duquel évaluer, dans l'espace et dans le temps, les pratiques d'aujourd'hui. Est ainsi posée la question de la prise en compte des interdépendances, spatiales et temporelles, des pratiques (Laganier et al, 2002)

7 Enfin, avec l'idée du développement durable, il s'agit d'une autre vision du développement basée davantage sur la recherche de solutions contextualisées et donc fortement diversifiées que sur la mise en place d'un modèle technique universel. Les indicateurs intermédiaires du développement durable sont, peut-être, en ce sens, plus des outils d'éclairage sur la diversité des solutions et de responsabilisation collective que des marqueurs de progrès vers un modèle.

8 La question du référentiel d'évaluation ne se pose pas que pour l'évaluation des pratiques. Elle se pose de façon aussi aiguë en ce qui concerne l'évaluation des politiques publiques. C'est sur ce dernier aspect que nous voulons insister maintenant.

9 La Loi d'Orientation agricole de 1999 associe à la notion de durabilité celle de multifonctionnalité 7 . Les pays de l'OCDE ont également lancé une réflexion commune sur ces deux thèmes en 1998 (OCDE, 2001a, 2001b) ${ }^{8}$. La question de la durabilité peut être 
associée à celle de la multifonctionnalité des activités agricoles, en particulier dans le sens où serait au fond durable une agriculture qui serait "plus» ou «mieux» multifonctionnelle. En effet, aujourd'hui, la capacité de l'agriculture à satisfaire les besoins actuels est autant prise en défaut que sa capacité à ne pas compromettre la satisfaction des besoins des générations futures. Autrement dit, la recherche d'une agriculture durable ne se justifie pas seulement par les atteintes à l'environnement (en ce qu'elles hypothèquent le renouvellement des ressources naturelles) mais aussi par le fait que les produits issus de l'agriculture ne correspondent plus complètement aux attentes des sociétés. Dans les sociétés «en développement», l'agriculture ne permet pas de satisfaire les besoins alimentaires de base, notamment en raison d'une concurrence considérée comme déloyale des agricultures des pays développés. Le principe d'équité intra-générationnelle, c'est-à-dire spatiale est ainsi pris en défaut et c'est un des motifs de la recherche d'une agriculture développée plus durable. De plus, dans les sociétés développées, l'insatisfaction se double d'une dimension qualitative. Plus précisément, d'une part les produits «de base», à usage alimentaire ou industriel ne présentent plus de manière indéfectible les garanties de sécurité sanitaire qu'exigent les consommateurs, et d'autre part les «produits-joints» à ces produits de base (aménités environnementales et sociales) sont parfois en décalage avec la demande sociétale - en notant que ces produits joints, pour l'essentiel, ne sont pas marchands et ont donc un caractère de bien public. Ce sont précisément ces derniers décalages entre offre et demande (considérées ici sous l'angle qualitatif) qui ont, d'une certaine façon, révélé la multifonctionnalité de l'agriculture.

10 Sans rentrer plus avant dans les débats sur la notion de multifonctionnalité ${ }^{9}$, nous souhaitons seulement attirer l'attention sur le fait que la définition de la multifonctionnalité met en évidence le caractère public des problèmes à traiter en matière de durabilité (Allaire, Dupeuble, 2003a). Prenons l'exemple simple de la contribution volontaire ou non d'un agriculteur à la qualité d'un paysage. Le caractère de bien public dudit paysage (en tant qu'objet esthétique) repose sur l'existence d'un site de vue permettant aux amateurs de l'admirer. Mais il ne suffit pas qu'une fois vu, ce paysage reste visible pour les autres (non exclusivité) sans altération (non rivalité) pour acquérir le statut de bien public. En effet, cette seule considération suppose que tout un chacun ait un intérêt égal pour ce paysage, i.e. il est d'intérêt public. Alors, le soutien à des activités menacées de disparition mais qui sont déterminantes de la qualité d'un paysage ou un programme local d'embellissement constituent de fait un paysage en bien public. Ce faisant ces actions introduisent les questions complémentaires de l'accès public à ce paysage et des modalités de définition des actions de restauration ou d'embellissement. Cette analyse est valable mutatis mutandis pour chacun des effets pris en compte dans le cadre de la multifonctionnalité. La qualification de certains effets d'activités comme étant des biens publics est liée à un jugement social. Ainsi l'imbrication des dimensions publique et privée de l'activité agricole apparaît clairement. Mais, les produits-joints à l'activité agricole, c'est-à-dire ces autres fonctions d'intérêt public, ne peuvent entrer dans le jeu économique (par des incitations marchandes ou venant des politiques publiques) que si on peut les circonscrire dans leurs effets, délimiter ceux-ci et les mesurer (Callon, 1999).

11 C'est dans le cadre des débats nécessaires à la circonscription des problèmes publics liés à la multifonctionnalité que les porte-parole des générations futures, s'ils existent, peuvent faire valoir les droits de ces dernières, donc que le temps long peut être intégré. C'est 
également dans ce cadre que peuvent être intégrées les différentes échelles spatiales concernées par les biens publics en jeu mais c'est en même temps ce qui en fait toute la difficulté. En effet, celle-ci tient précisément au fait que les effets externes de l'activité agricole concernent à la fois des biens publics locaux et globaux (pour la définition de ces notions, voir Kaul, 1999). Les biens publics locaux sont représentés, par exemple, par des accords conclus à l'échelle d'une commune pour entretenir des chemins d'exploitation tant pour l'usage agricole qu'aux fins de randonnées (pédestres, équestres ou encore cyclistes) dans le cadre de plateformes multi-usages. Mais dans le cas des biens environnementaux dédiés aux générations futures (contrôle de l'effet de serre, biodiversité...), comme dans celui de la fourniture d'une nourriture saine et suffisante, ou encore de marchés efficaces, de la sécurité et de la paix mondiale, etc., le caractère universel commun à ces différents biens leur confère une dimension globale. Toutefois, bien que globaux, ces biens publics ne reposent pas moins sur des conditions locales: la biodiversité planétaire est bien la résultante des biodiversités locales. En fait, ces biens publics résultent finalement de dynamiques sociales complexes dans lesquelles interfèrent largement sphère privée et sphère publique, dans le temps et l'espace.

Les économistes ou autres évaluateurs sont donc confrontés à la nécessité de décomposer l'analyse entre le niveau local et global. Ainsi, par exemple, Randall (2002) souligne que la tâche d'évaluation des produits globaux de l'agriculture, vue comme multifonctionnelle, «dépasse en ampleur ce qui a été tenté jusque là», bien au-delà de ce que peut imaginer la communauté scientifique de l'évaluation environnementale, précise-t-il. Il propose finalement un schéma d'évaluation à deux niveaux: celui, global, du consentement à payer pour l'environnement par les citoyens (payeurs d'impôts) et celui des "prix verts", c'est-à-dire de la valeur à attribuer aux fonctions environnementales, qui "sont contextuels et comportent un grand nombre d'éléments et doivent être estimés à une échelle nationale ou continentale, mais déterminés ferme par ferme". Dans ce schéma de l'évaluation, nous pensons qu'il est nécessaire d'introduire des niveaux intermédiaires ou "collectifs", en particulier territoriaux.

L'activité individuelle des producteurs repose sur des ressources collectives, particulièrement décisives en agriculture du fait de l'interdépendance technique des exploitations sur un territoire et des interdépendances économiques dans un bassin de production. De ces interdépendances résulte le caractère collectif de fait des produitsjoints de l'activité agricole ; ce qui, en fin de compte, leur donne le statut de biens publics intermédiaires. La haie n'a de valeur paysagère ou biologique que si elle contribue à la constitution d'un bocage lui-même reconnu pour sa valeur paysagère et biologique. Ces interdépendances n'ont pas un caractère permanent et universel, elles résultent de facteurs techniques et sociaux et sont à l'origine de dispositifs de coopération entre producteurs. Que ces coordinations viennent à faillir et c'est la multifonctionnalité et sa contribution au développement durable qui sont remises en cause.

14 L'objectif d'améliorer la multifonctionnalité des exploitations agricoles peut s'interpréter, en matière de politique publique, comme la volonté de restaurer des complémentarités locales ou d'en susciter de nouvelles; il s'agit d'inciter à la négociation de plates-formes d'usage partagé des ressources naturelles qui sont de gestion locale. Dans cette perspective, l'évaluation de la durabilité au seul niveau de l'exploitation ne permet pas de mesurer ne serait-ce que l'existence de ces coordinations. Une analyse à des niveaux supérieurs, territoriaux, est indispensable. Mais quel est le bon niveau? Pour peu que l'on considère l'insertion d'une activité dans le temps et l'espace, on est 
rapidement confronté à de nombreuses incertitudes. Ce qui caractérise, dans la littérature spécialisée, des biens publics "globaux" (mais qui ne sont en général définis qu'en tant que biens "intermédiaires", comme nous l'avons vu), c'est que les bénéfices (ou les inconvénients, s'ils s'agit d'effets négatifs) en sont largement dispersés dans l'espace et dans le temps. La production de ces effets fait intervenir d'une manière ou d'une autre différents types de stocks: des stocks de polluants, de connaissances, des stocks biologiques ou génétiques... Lorsque les effets externes d'une activité (ou externalités) affectent un de ces stocks, on peut les qualifier «d'externalités de stocks» car leur manifestation n'est pas directe mais fonction de l'importance du stock qu'elles affectent: l'émission d'un polluant (la matière active d'un désherbant) dans l'environnement se manifeste en fonction du stock initial de ce polluant (le niveau initial de concentration de cette matière active dans les eaux d'une nappe phréatique). Les externalités de stock ont des conséquences durables, irréversibles ou quasi irréversibles, mais très largement imprévisibles, comme le montrent de nombreux problèmes d'environnement. De ce point de vue, l'activité agricole, par les ressources qu'elle mobilise et par ses produits, n'est pas en relation avec un territoire que l'on pourrait délimiter mais avec plusieurs, selon la fonction que l'on considère. D'un point de vue économique, les relations territoriales d'une exploitation agricole peuvent être analysées comme des "externalités de stock". Une exploitation entretient un stock de fertilité (le sol), des stocks de polluants, dispersés et diffus, des stocks de savoir (dans des groupes professionnels), etc. C'est ainsi qu'elle s'inscrit dans des processus globaux. Autrement dit, plus le problème est vu globalement, en prenant en compte les effets à la fois reportés dans l'espace ou dans le temps et liés à des phénomènes cumulatifs, plus les éléments d'analyse du problème deviennent imprécis et incertains et plus interfèrent les différents registres (privé, collectif et public) de la régulation (Allaire, 2003). On sera donc amené d'une part à rechercher les espaces pertinents pour l'analyse des interdépendances (via les effets de stock comme cela a été précisé) et d'autre part les espaces où se déploient des actions régulatrices, qui ne coïncident pas nécessairement.

Dans une optique d'évaluation des politiques publiques territoriales ${ }^{10}$, pour contourner les obstacles méthodologiques, il est possible de dissocier les questions de la détermination des enjeux et celle de leur mesure dans diverses sortes de territoires. Pour dégager des indicateurs de multifonctionnalité et de durabilité, il convient de produire à la fois des indicateurs permettant de mesurer des enjeux territoriaux dans différents domaines environnementaux et sociaux, et des indicateurs permettant de mesurer l'adaptation des activités à ces enjeux. Par exemple, les objectifs visés par le dispositif des contrats territoriaux d'exploitation (CTE) sont des biens publics qui se situent à un niveau élevé de globalité: "Préserver et améliorer la qualité de l'eau", "Lutter contre l'érosion", "Maintenir et créer de l'emploi", etc. La complexité des voies techniques par lesquelles ces objectifs sont atteints implique que la mesure de la contribution de chaque source élémentaire aux biens finalement attendus n'est pas immédiate, mais repose elle-même sur de nombreux intermédiaires. Dans cette perspective, des indicateurs tels que le taux d'adoption de CTE centrés sur la problématique de l'eau dans des territoires constitués au regard de cette problématique (en croisant par exemple le zonage découlant de la directive nitrates et celui de la qualité des eaux tel qu'on peut l'élaborer à partir des mesures réalisées par les agences de l'eau) peuvent être considérés comme des indicateurs de durabilité du développement des territoires et des activités. Toutefois, de tels indicateurs demandent à être adaptables aux évolutions des enjeux et des situations. 

des territoires d'action et d'évaluation) doivent être pris en compte dans l'élaboration d'indicateurs concernant les politiques publiques. Par exemple, des indicateurs permettant de qualifier le degré de variété et d'implication des différentes parties prenantes de tel ou tel enjeu dans la circonscription des problèmes et le choix des actions à conduire sont également révélateur de la durabilité des dynamiques de développement. En effet, complexité des problèmes, interdépendance local/global, et incertitude demandent à l'évidence une adaptation des procédures délibératives comme condition d'une gouvernance durable (Laganier et al, 2002).

L'impératif du développement durable fait de la question de l'intégration des enjeux économiques, environnementaux et sociaux du développement une question majeure. Il impose d'appréhender les phénomènes à différentes échelles spatio-temporelles. Il est généralement considéré qu'il reste du chemin à parcourir pour donner aux divers indicateurs qui ont été proposés une flexibilité suffisante pour s'adapter à une variété de configurations territoriales (IFEN, 2002). Certes, on peut multiplier les indicateurs à différentes échelles, voire modéliser certaines des relations entre différents niveaux de décision. Toutefois, il y a des obstacles épistémologiques (ou cognitifs) aux changements d'échelles. En effet, nous sommes en présence de co-évolutions de différents ordres biophysiques et socio-économiques et non de relations de détermination.

Cependant, les incertitudes et les difficultés à définir le développement durable ne sont pas que d'ordre méthodologique. L'évolution de nos sociétés vers ce que l'on appelle des "sociétés de la connaissance" ne se caractérise pas seulement par le rôle que joue l'expertise scientifique dans les questions publiques, mais tout autant par la circulation des signes et des opinions. La diversité des modes de production ne peut être appréhendée au travers d'une technique idéale, où les pratiques seraient scientifiquement adaptées à la variété des conditions écologiques. Potentiellement infinie, cette variété n'est toujours que partiellement connue et, qui plus est, elle n'est connue qu'au travers des relations techniques. Différents modes de produire ont également diverses légitimités éthiques, qui introduisent d'autres modes de connaissance des objets. Cette diversité produit un monde global polymorphe et conflictuel, en même temps que la notion de développement durable appuie l'émergence d'un régime de responsabilité publique (accountability). C'est dans cette perspective que la construction des indicateurs du développement durable est lui-même un problème public.

\section{BIBLIOGRAPHIE}

Allaire G., 2003. Régulation et Gouvernance. Actes des Journées AISLF à Rouen, 12/09/2002.

Cellule Gris $n^{\circ} 9$, juin 2003, pp. 37-62.

Allaire G., Dupeuble T., 2003a. De la multifonctionnalité de l'agriculture à la multi-évaluation de la production agricole, Economie Rurale, $n^{\circ} 275$, juin 2003.

Développement durable et territoires , Varia (2004-2010) | 2004 
Allaire G., Dupeuble T., 2003b. De la multifonctionnalité de l'activité agricole à la multiévaluation de la production agricole: Vers l'émergence d'un nouveau système de compétences. Actes du colloque de la SFER "La multifonctionnalité de l'activité agricole et sa reconnaissance par les politiques publiques", 21 et 22 mars 2002, Paris. Educagri, SFER, CIRAD, éditeurs, pp. 195-251.

Bruntland G. ,1989. Notre avenir à tous, Commission mondiale sur l'environnement et le développement, Ed. du Fleuve, Montréal.

Callon M., 1999. La sociologie peut-elle enrichir l'analyse économique des externalités? Essai sur la notion de cadrage-débordement, dans Innovation et performance: Approches interdisciplinaires, sous la direction de Foray D. et Mairesse J., éditions EHESS, Paris.

Hubert B., Godart O., 2002. Le développement durable et la recherche scientifique à l'Inra, Rapport à Mme la Directrice générale de l'Inra.

IFEN, 2001. Propositions d'indicateurs de développement durable pour la France. Collection «Etudes et travaux», $\mathrm{n}^{\circ}$ 35, Novembre 2001.

Kaul I., 2001. Public Goods: Taking the Concept to the 21st Century, in The Market of the Public Domain Drache D. (ed.), London and New York: Routledge, pp. 255-273.

Landais E., 1999. Agriculture durable: les fondements d'un nouveau contrat social?, Courrier de l'environnement de l'Inra, n³3, avril 1999

Laganier R., Villalba B., ZuindeauB., 2002. Le développement durable face au territoire: éléments pou une recherche pluridisciplinaire, Dossier «Approches territoriales du développement durable», e-revue Développement durable et territoires, www.revue-ddt.org.

Lepage P, 1997. Communication à l'assemblée générale du CEDAG, Rennes, mai 1997

OCDE, 2001a.Développement durable. Quelles politiques.

OCDE, 2001b. Multifunctionality: towards an analytical framework, COM/AGR/APM/TD WP.

Randall A., 2002. Valuing the outputs of multifunctional agriculture, European Review of Agricultural Economics, Vol 29 (3), pp. 289-307.

Références des différentes grilles d'évaluation:

La méthode IDEA, Lionel Vilain, Educagri éditions, collection Approches, 2000. http:// www.editions.educagri.fr/publication/detail.cfm?code=DD2004

Evaluer la durabilité d'un système de production: Approche, méthodes, diagnostics. Réseau agriculture durable, collection Cahiers techniques de l'agriculture durable, Impact Bretagne, Rennes, 2000.

Les cahiers techniques de l'agriculture durable, Réseau agriculture durable, 2001.

http://www.agriculture-durable.org/

DIALECTE, Solagro, Toulouse.http://www.solagro.org/site/014.html

\section{NOTES}

1. Dans cette optique, voir notamment le rapport remis par B. Hubert et O. Godard à Mme la Directrice de l'INRA. Les auteurs notent que cet élargissement pose de redoutables problèmes à la recherche, dont la démarche classique consiste à isoler des phénomènes 
dans un contexte stabilisé et maîtrisé afin de mieux les caractériser, alors qu'il s'agit ici de les appréhender dans un contexte où ils sont définis par des procédures faisant appel à d'autres acteurs que ceux du monde "scientifique".

2. Une première présentation en a été faite au séminaire agriculture durable d'AGROMIP (Toulouse), le 3 juillet 2003.

3. Forum pour une agriculture raisonnée respectueuse de l'environnement.

4. Centres d'initiatives pour valoriser l'agriculture et le milieu rural.

5. Ce réseau rassemble les exploitations agricoles intégrées aux lycées agricoles parties prenantes d'une action nationale menée dans le cadre du volet formation des mesures agri-environnementales du règlement 2078/92.

6. Toutefois, s'il s'agissait au départ d'initiatives associatives, on voit aujourd'hui se mettre en place une certification des exploitations agricoles. Ainsi la notion d'agriculture «raisonnée» a aujourd'hui un fondement légal avec le décret n 2002-631 du 25 avril 2002 relatif à la qualification des exploitations agricoles au titre de l'agriculture raisonnée.

7. LOA 1999, Article $n^{\circ} 1$ : «La politique agricole prend en compte les fonctions économique, environnementale et sociale de l'agriculture et participe à l'aménagement du territoire, en vue d'un développement durable».

8. Pour l'OCDE, le développement durable a pour objectif de «assurer un progrès économique équitable sur le plan social, tout en préservant la base de ressources et l'environnement pour les générations futures» (OCDE, 2001a). La multifonctionnalité, d'un point de vue normatif, renvoie elle, en général, à un argument justifiant une certaine protection de l'agriculture au regard des biens publics territoriaux (localisés) qu'elle fournit, mais il n'y a pas de consensus entre les pays sur les implications en matière de politique agricole. L'approche de la multifonctionnalité dans les travaux de l'OCDE (2001b) renvoie à une définition «positive»: L'agriculture est multifonctionnelle en ce qu'elle produit, de manière jointe, des produits de base (à vocation alimentaire ou industrielle), et des produits «autres», lesquels ont un caractère d'externalité ou de bien public. En même temps que l'agriculture produit du blé, utilisé dans l'alimentation ou dans des filières industrielles (amidon), elle produit du paysage, de la biodiversité, voire de l'emploi, etc. C'est du caractère joint de ces produits que découle la multifonctionnalité. Plus fondamentalement, c'est du caractère a priori multifonctionnel des actifs que découle la multifonctionnalité des activités. Pour une analyse plus détaillée des débats autour de la notion de multifonctionnalité, voir Allaire et Dupeuble (2003 a et b).

9. Un des points d'achoppement des débats internationaux sur la question de la multifonctionnalité a été la question de l'emploi: Est-ce que les emplois induits par une activité dans un territoire sont à considérer comme une "fonction" de cette activité? Du point de vue du développement durable, la répartition des emplois est un critère à prendre en compte, mais qui ne se définit pas au niveau local.

10. La notion de politique territoriale peut recouvrir tant des politiques d'initiative locale que des politiques de niveau national ou européen répondant à des enjeux territorialisés. Une évolution de la conception des politiques publiques conduit à une territorialisation croissante de l'action publique par la différenciation des modalités de sa mise en œuvre selon les territoires auxquels elle s'applique. 


\section{RÉSUMÉS}

L'opérationnalisation du concept d'agriculture durable au moyen de la production d'indicateurs applicables à l'exploitation agricole pose de nombreuses difficultés en raison de l'intrication des dimensions spatiale et temporelle des problèmes et pratiques en jeu: les changements d'échelle sont alors problématiques. Les problèmes à traiter dans la perspective de plus de durabilité, compte tenu de leur nature publique, demandent d'être circonscrits à plusieurs niveaux: outre les niveaux global (biens publics mondiaux) et local (activités privées), un niveau intermédiaire collectif et territorial est indispensable pour l'élaboration de solutions socialement acceptables.

To deal with «sustainable agriculture» in practical ways needs to set up indicators. But such indicators are quite difficult to find out, due to the multi-scaled and inter-temporal dimensions of external effects of economic activities and especially of agricultural practices. Externalities issues, which are problems characterized by their public nature, have to be defined and sized at different levels: besides the global one (which concerns debates on global public goods) and the local one (how sustainable are private activities), collective and territorial levels are also necessary to set up socially acceptable responses.

\section{INDEX}

Keywords : agriculture

Mots-clés : durabilité, indicateurs, multifonctionnalité, territoire, biens publics 